\title{
Molecularly imprinted titania nanoparticles for selective recognition and assay of uric acid
}

\author{
Adnan Mujahid • Aimen Idrees Khan • Adeel Afzal • \\ Tajamal Hussain • Muhammad Hamid Raza • \\ Asma Tufail Shah $\cdot$ Waheed uz Zaman
}

Received: 26 June 2014/ Accepted: 5 August 2014/Published online: 26 August 2014

(C) The Author(s) 2014. This article is published with open access at Springerlink.com

\begin{abstract}
Molecularly imprinted titania nanoparticles are successfully synthesized by sol-gel method for the selective recognition of uric acid. Atomic force microscopy is used to study the morphology of uric acid imprinted titania nanoparticles with diameter in the range of $100-150 \mathrm{~nm}$. Scanning electron microscopy images of thick titania layer indicate the formation of fine network of titania nanoparticles with uniform distribution. Molecular imprinting of uric acid as well as its subsequent washing is confirmed by Fourier transformation infrared spectroscopy measurements. Uric acid rebinding studies reveal the recognition capability of imprinted particles in the range of $0.01-0.095 \mathrm{mmol}$, which is applicable in monitoring normal to elevated levels of uric acid in human blood. The optical shift (signal) of imprinted particles is six times higher in comparison with non-imprinted particles for the same concentration of uric acid. Imprinted titania particles have shown substantially reduced binding affinity toward interfering and structurally related substances, e.g. ascorbic acid and guanine. These results suggest the possible
\end{abstract}

A. Mujahid $(\bowtie) \cdot$ A. I. Khan · T. Hussain •

M. H. Raza · W. uz Zaman

Institute of Chemistry, University of Punjab, Quaid-e-Azam

Campus, Lahore 54590, Pakistan

e-mail: adnanmujahid.chem@pu.edu.pk

\section{A. Afzal $(\square)$}

Affiliated Colleges at Hafr Al-Batin, King Fahd University of Petroleum and Minerals, P.O. Box 1803, Hafr Al-Batin 31991, Saudi Arabia

e-mail: aa@aafzal.com

\section{A. Afzal · A. T. Shah}

Interdisciplinary Research Centre for Biomedical Materials, COMSATS Institute of Information Technology, Defence Road, Off. Raiwind Road, Lahore 45600, Pakistan application of titania nanoparticles in uric acid recognition and quantification in blood serum.

Keywords Molecular imprinting · Nanoparticles · Spectroscopy $\cdot$ Titania $\cdot$ Uric acid

\section{Introduction}

Molecular imprinting (Mosbach 1994; Chen et al. 2011) is a modern technique to create artificial receptor sites in a variety of materials such as polymers (Latif et al. 2011), nanoparticles (Lieberzeit et al. 2007), and others. In the last decade, molecular imprinting has proven itself a promising technology to build materials with several applications in analytical separations (Kempe and Mosbach 1995; Andersson 2000), enzyme-like catalysis (Ramström and Mosbach 1999; Wulff 2002), chemical sensors (Dickert and Hayden 1999; Mujahid et al. 2010b), and advance drug delivery systems (Hilt and Byrne 2004; Sellergren and Allender 2005; Kryscio and Peppas 2009). Molecularly imprinted polymers (MIPs) are robust, less expensive, can endure high temperature and oxidative environment, and are chemically stable as compared to their natural competitors (Vasapollo et al. 2011). Molecularly imprinted nanoparticles (MINPs), on the other hand, possess high surface area and a large number of interaction sites in addition to the aforementioned attributes of MIPs (Iqbal and Afzal 2013).

In general, imprinting can lead to fast and easy production of enzyme- or antibody-like binding sites (Poma et al. 2010; Mujahid et al. 2013), thus proving a potential breakthrough technique in the field of biomedical materials and clinical diagnostics (Piletsky et al. 2006). Imprinted materials can be synthesized by a variety of methods like suspension, emulsion polymerization (Pérez et al. 2001) and precipitation (Ye 
et al. 2000) and others to prepare MIPs directly (Ansell and Mosbach 1998; Zhang et al. 2003; Chen et al. 2005). Among various imprinting approaches, non-covalent imprinting is more advantageous due to simple and straightforward synthetic procedure, commonly available starting materials and chemicals, and relatively easy extraction of weekly bounded template molecules (Sellergren 2000; Zhang et al. 2006).

Uric acid is a final product of purine metabolism in human body (Kaur and Halliwell 1990). Elevated levels of uric acid can cause crystal formation in the joints, which is known as gout. It is reported that half of the antioxidant capacity of blood plasma in human comes from uric acid (Maxwell et al. 1997). The normal range of uric acid in human blood plasma is between 3.6 and $8.3 \mathrm{mg} \mathrm{dL}^{-1}$, or 0.214 and $0.493 \mathrm{mmol} \mathrm{L}^{-1}$. The elevated level of uric acid in blood may result in the formation of kidney stones, i.e. urate crystallizes in the kidney. These stones are radiolucent and do not appear in an abdominal plain X-ray scan, so their presence must be detected by ultrasound.

The main focus of this research is to generate molecularly imprinted titania nanoparticles for the selective recognition of uric acid. Since titania is resistive to oxidation and degradation processes (Mujahid et al. 2010a), therefore, it is suitable for working in complex matrices with imprinted affinity sites for uric acid. Since molecular imprinting accomplishes the desired affinity for target analyte molecules. Although, there are several reports focused on the detection and assay of uric acid using different types of sensing interfaces (Patel et al. 2009; Sun et al. 2011; Khasanah et al. 2012; Wang et al. 2013), imprinted nanomaterials such as metal oxide nanoparticles have not been considered for this purpose so far. Therefore, it would be interesting to develop highly sensitive and chemically stable recognition materials that can readily detect and monitor uric acid in blood plasma. This article presents our primary results showing the potential of imprinted titania nanoparticles toward real-time monitoring of uric acid in blood plasma.

\section{Experimental section}

\section{Chemicals and reagents}

All the chemicals and reagents used were obtained as follows: Titanium butoxide, uric acid, and iso-propanol from Sigma Aldrich, sodium hydroxide from Merck and methanol from United Laboratory Chemicals.

\section{Characterization methods}

Morphology of the imprinted titania nanoparticles is studied by Nanoscope III Atomic Force Microscope. Scanning electron microscopy (SEM) image of titania nanoparticles' film was recorded on Hitachi S-4700 Scanning Electron Microscope. The microscopic images were analyzed using WSxM v5.0 by NANOTEC Electronica (Horcas et al. 2007). Fourier transformation infrared spectroscopy (FTIR) spectra were recorded on Bruker Vector 22 FTIR Spectrometer. UV-vis spectroscopy was performed on Labomed Spectrophotometer model UVD-2950.

\section{Synthesis of molecularly imprinted titania nanoparticles}

Molecularly imprinted titania $\left(\mathrm{TiO}_{2}\right)$ nanoparticles were synthesized by sol-gel method using titanium butoxide as a precursor. A solvent system for dissolving uric acid was designed comprising of $60 \%$ methanol and $40 \% 0.1 \mathrm{~N}$ $\mathrm{NaOH}$. Precisely, $0.284 \mathrm{~g}$ of titanium butoxide was dissolved in $10 \mathrm{~mL}$ of $i s o$-propanol taken in a reaction flask placed on hot plate at $60{ }^{\circ} \mathrm{C}$ under $130 \mathrm{rpm}$. Following that $0.005 \mathrm{~g}$ of uric acid was dissolved in a $5 \mathrm{~mL}$ of the abovementioned solvent and then added to above reaction mixture under constant stirring. The hydrolysis of titanium butoxide was carried out by the basic media of uric acid solvent. The nanoparticles started to form slowly. The stirring was kept on for almost $1 \mathrm{~h}$. Then stirring and heating were turned off and the particles were subjected to centrifugation for $10 \mathrm{~min}$ at $5,000 \mathrm{rpm}$. Finally, the supernatant was removed and particles were dried in oven for maximum $1 \mathrm{~h}$ at $120^{\circ} \mathrm{C}$. As-prepared nanoparticles were washed with excess of solvent to remove any traces of template, i.e. uric acid present in them, and then were characterized for the successful removal of uric acid. Nonimprinted titania particles were also prepared following the same procedure except adding uric acid.

\section{Re-inclusion/rebinding studies}

Re-inclusion or rebinding studies of molecularly imprinted titania nanoparticles were carried out for different concentrations of uric acid as in human blood from normal to elevated levels. For binding studies, $0.02 \mathrm{~g}$ of thoroughly washed imprinted titania nanoparticles was added to uric acid solution of concentration $0.1 \mathrm{mmol}$ of volume $20 \mathrm{~mL}$ under $130 \mathrm{rpm}$ for $1 \mathrm{~h}$ at room temperature. After stirring, the mixture was centrifuged for $10 \mathrm{~min}$ at 5,000 rpm and the supernatant was separated and analyzed by spectrophotometer. The absorbance of uric acid solution before and after adding the particles was monitored and used for calculating static adsorption coefficient. The procedure was repeated for other concentrations of uric acid starting from 0.01 to $0.095 \mathrm{mmol}$. This concentration range is about ten folds lower for both normal and elevated levels of uric acid in human blood respectively. The purpose of selecting such low concentration range for uric acid was to minimize the 
matrix effects of blood serum, i.e. non-specific sorption. The non-imprinted titania nanoparticles and other interfering analytes were also tested in the same manner.

\section{Results and discussion}

\section{Morphological characterization}

Microscopic characterization of molecularly imprinted titania nanoparticles was performed via Atomic force microscopy (AFM) and SEM to study their morphology. The two- and three-dimensional AFM images of as-prepared or imprinted titania nanoparticles are shown in Fig. 1. AFM reveals the morphology, i.e. size and shape of titania nanoparticles. The AFM image of powdered titania sample suggests that imprinted titania nanoparticles are spherical in shape with an average particle size or diameter in the range of 100-150 nm. Moreover, these nanoparticles are not coalesced to form bigger aggregates and size distribution is homogeneous. It may be due to the presence of uric acid - the template attached to the surface of imprinted titania nanoparticles-that may prevent coalescence of these particles.

Figure 2 shows the SEM image and 3D surface construct of a thick film of imprinted titania nanoparticles formed by coating a dense paste of powdered titania on quartz crystal. The microstructure and surface morphology of imprinted nanoparticles is obvious. These images demonstrate that thick films of titania nanoparticles can be fabricated easily leading to the formation of a very fine network of imprinted titania nanoparticles. Since, each of these imprinted titania particles has template-specific interaction sites, the dispersion of titania nanoparticles in thick film ensures that these interaction sites are homogeneously distributed. The high number of interaction centers is complementary for high sensitivity and shorter adsorption pathways.
Spectroscopic characterization

Fourier transformation infrared spectroscopy measurements of uric acid imprinted titania nanoparticles, i.e. asprepared, and molecularly imprinted but thoroughly washed titania nanoparticles as well as non-imprinted particles were performed to examine the imprinting characteristics and any pronounced differences in chemistry and functionality of these nanoparticles. The FTIR spectra of different types of titania nanoparticles are also shown in Fig. 3. The predominant functional groups (peaks) observed in different types of titania nanoparticles are recorded in Table 1.

As shown in Fig. 3, the characteristic amide's $\mathrm{C}=\mathrm{O}$ stretching vibrations and secondary amine's $\mathrm{C}-\mathrm{N}$ stretching vibrations appear at 1,673 and $1,290 \mathrm{~cm}^{-1}$, respectively (Mohan 2004). It is obvious that these groups are present only in case of as-prepared uric acid imprinted titania nanoparticles (before washing), since these functional groups originate from uric acid molecules. Thus, repeated washing of these nanoparticles successfully removes the template molecules leading to the absence of carbonyl and amine peaks in imprinted and thoroughly washed titania nanoparticles. Expectedly, non-imprinted titania particles do not indicate any such absorption for amide and amine groups.

However, the presence of Ti-O group can be confirmed in all three types of nanoparticles from the broad absorption in the range of $600-750 \mathrm{~cm}^{-1}$ (Rubab et al. 2014). The IR absorption of alcoholic group (TiO-H) indicates the formation of titania networks with sufficient number of surface hydroxyl groups, which may lead to weak interactions, e.g. hydrogen bonding, between the particles and the template molecules. Moreover, TiO-H absorption of as-prepared titania is different from the washed imprinted and non-imprinted particles due to the presence of amine $(\mathrm{N}-\mathrm{H})$ groups in molecularly imprinted titania nanoparticles.
Fig. 1 Two-dimensional (a), and three-dimensional (b) atomic force micrographs of as-prepared uric acid imprinted titania nanoparticles
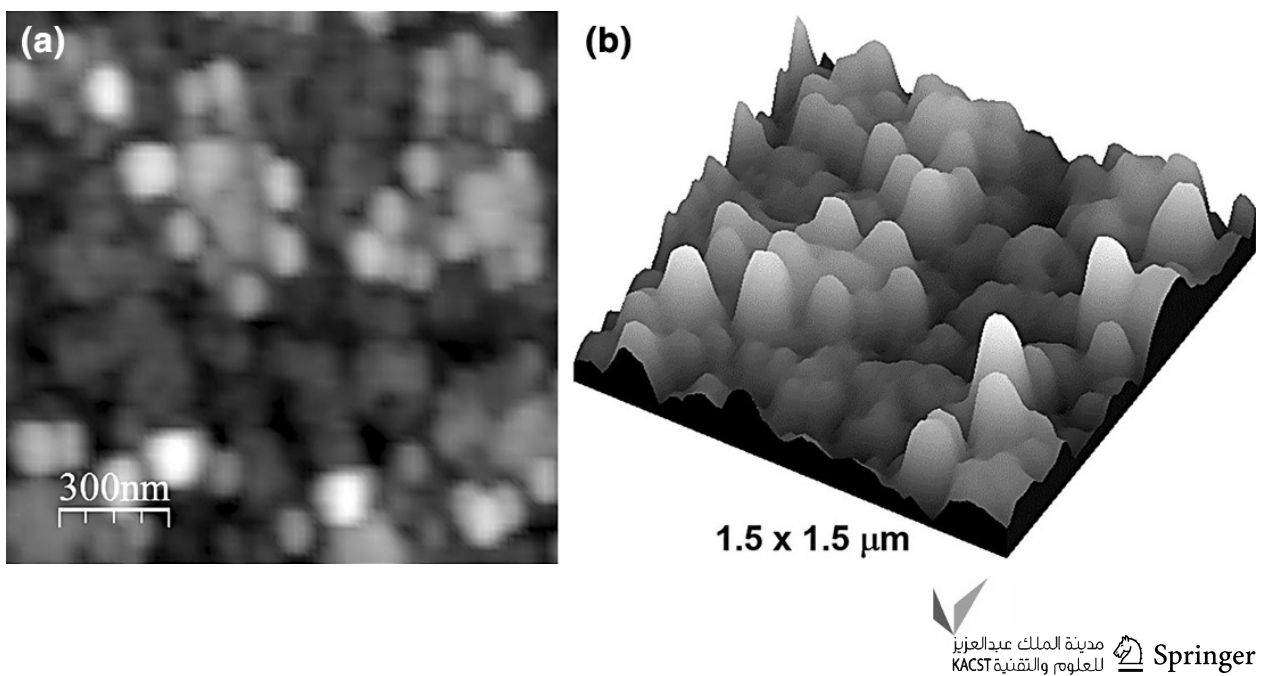

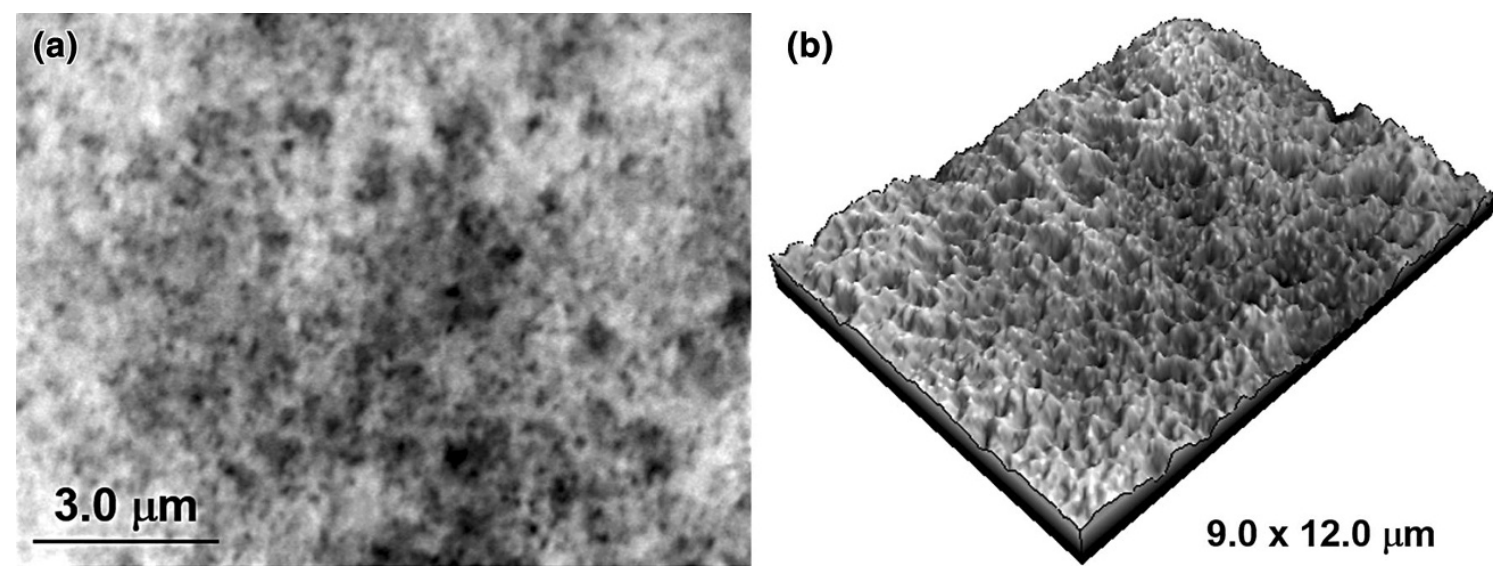

Fig. 2 The SEM image of molecularly imprinted titania nanoparticles (a), and corresponding 3D surface construct (b) to shown surface morphology of titania nanoparticles

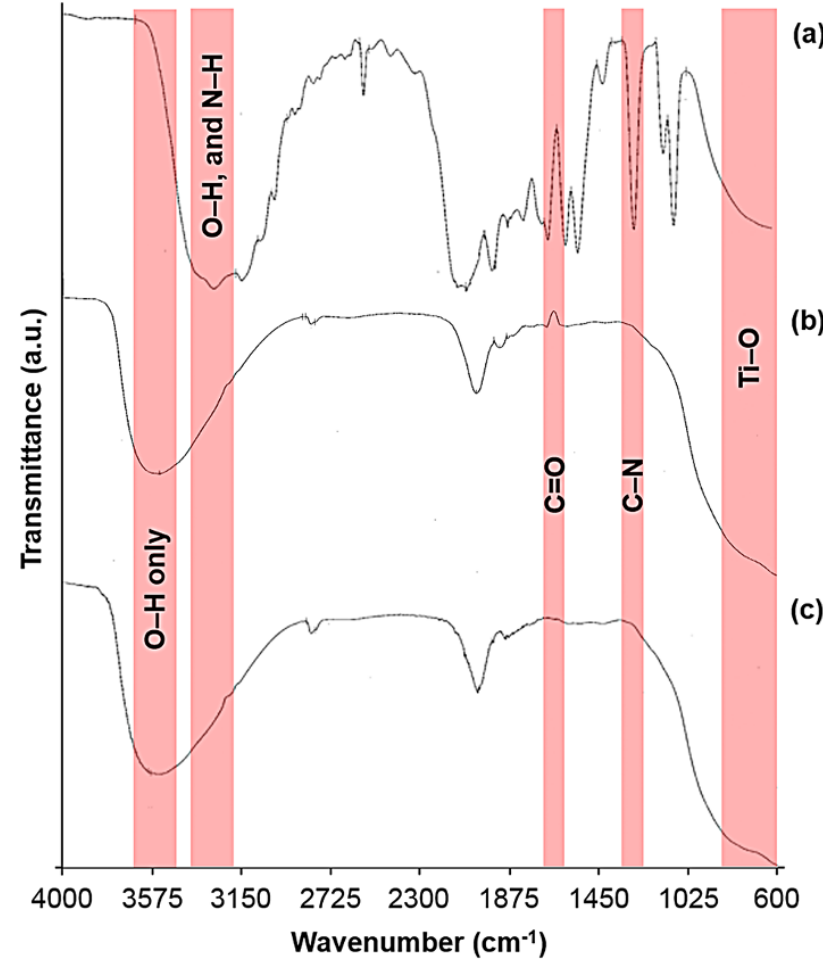

Fig. 3 FTIR spectra of as-prepared uric acid imprinted titania nanoparticles $(a)$, imprinted titania nanoparticles after washing and removal of template $(b)$, and non-imprinted titania nanoparticles $(c)$

Uric acid reinclusion/rebinding studies

The characteristics of uric acid imprinted titania nanoparticles such as selectivity and their binding capacity after complete template removal in the process of washing were studied by UV-vis spectrophotometric analysis. Thorough washing of molecularly imprinted titania nanoparticles removes the template, i.e. uric acid from the particulate matrix. Consequently, the absorbance is reduced after each washing step, as shown in Fig. 4.
Table 1 FTIR results of uric acid imprinted, washed, and nonimprinted titania nanoparticles

\begin{tabular}{llll}
\hline $\begin{array}{l}\text { Sample } \\
\text { nanoparticles }\end{array}$ & $\begin{array}{l}\text { Amine } \\
(\mathrm{C}-\mathrm{N}) \\
\left(\mathrm{cm}^{-1}\right)\end{array}$ & $\begin{array}{l}\text { Amide } \\
(\mathrm{C}=\mathrm{O}) \\
\left(\mathrm{cm}^{-1}\right)\end{array}$ & $\begin{array}{l}\text { Alcohols } \\
(\mathrm{Ti}-\mathrm{OH}) \\
\left(\mathrm{cm}^{-1}\right)\end{array}$ \\
\hline $\begin{array}{l}\text { Uric acid imprinted } \mathrm{TiO}_{2} \\
\begin{array}{l}\text { Imprinted } \mathrm{TiO}_{2} \text { after } \\
\text { washing }\end{array}\end{array}$ & - & $\begin{array}{l}1,673(\mathrm{sh}) \\
1,673(\mathrm{wh})\end{array}$ & $\begin{array}{l}3,290(\mathrm{~s}, \mathrm{~b}) \\
3,555(\mathrm{~b})\end{array}$ \\
\begin{tabular}{l} 
Non-imprinted $\mathrm{TiO}_{2}$ \\
\hline
\end{tabular} & - & - & $3,560(\mathrm{~b})$ \\
\hline
\end{tabular}

FTIR absorption intensity is denoted as $s h$ sharp, $s$ strong, $b$ broad; and $w$ weak

Subsequently, the rebinding of uric acid molecules in (and with) imprinted titania particles was studied through re-inclusion of analyte (uric acid) molecules. For this purpose, $0.02 \mathrm{~g}$ of imprinted titania nanoparticles was immersed in uric acid solutions of varying concentrations (ranging from 0.01 to $0.095 \mathrm{mmol}$ ) for $1 \mathrm{~h}$. After which, the mixture was centrifuged and the supernatant was analyzed on a UV/vis spectrophotometer. The re-inclusion of uric acid in imprinted titania nanoparticles was monitored by recording the differences in the absorbance of uric acid solutions before and after immersing titania nanoparticles. A calibration curve can be plotted from the differences in the absorbance of uric acid solution at various concentrations before and after rebinding (i.e. immersing titania nanoparticles for adsorption and re-inclusion of uric acid), as shown in Fig. 5.

The exact concentrations of uric acid solutions before and after its re-inclusion in imprinted titania nanoparticles were determined to calculate adsorption capacity of titania particles as described below. The quantity of uric acid adsorbed by the imprinted titania nanoparticles could also be calculated from the following equation.

$Q=\left(C_{\mathrm{i}}-C_{\mathrm{f}}\right) V / m$ 


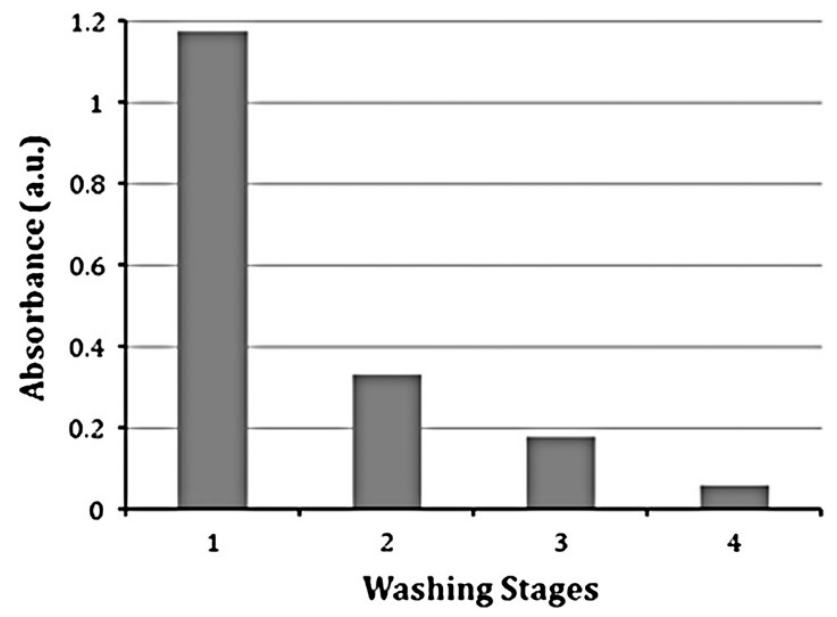

Fig. 4 Gradual decrease in absorbance of uric acid after each washing step: titania nanoparticles were washed repeatedly until no signal of uric acid was observed

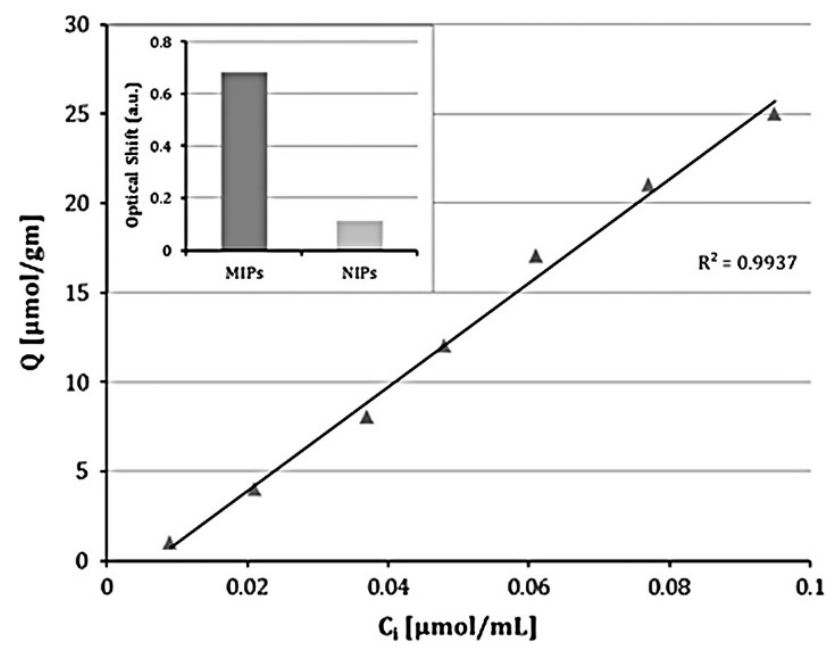

Fig. 5 Representation of adsorption isotherm for uric acid. Inset shows the bar graph for uric acid absorption by molecularly imprinted and non-imprinted titania nanoparticles

where, $C_{\mathrm{i}}$ and $C_{\mathrm{f}}$ are initial and final concentrations of uric acid solution expressed in $\mu \mathrm{mol} \mathrm{mL} \mathrm{m}^{-1}, V$ is the volume in $\mathrm{mL}$, and $m$ is the mass of imprinted particles taken in $\mathrm{g}$. So, the unit of $Q$ is $\mu \mathrm{mol} \mathrm{g}^{-1}$. A graph was plotted between $C_{\mathrm{i}}$ and $Q$ to interpret the binding affinity of the imprinted titania nanoparticles with the increasing concentration of uric acid, as shown in Fig. 5. In this way, it is clearly demonstrated that molecular recognition ability of imprinted titania nanoparticles increases linearly with the increasing concentration of uric acid.

In order to determine the non-specific interactions, both imprinted nanoparticles (MINPs) and non-imprinted nanoparticles (NINPs) were separately immersed in $1 \mathrm{mmol}$ solution of uric acid in binding experiments. The inset bar graph in Fig. 5 shows the relative optical shifts of

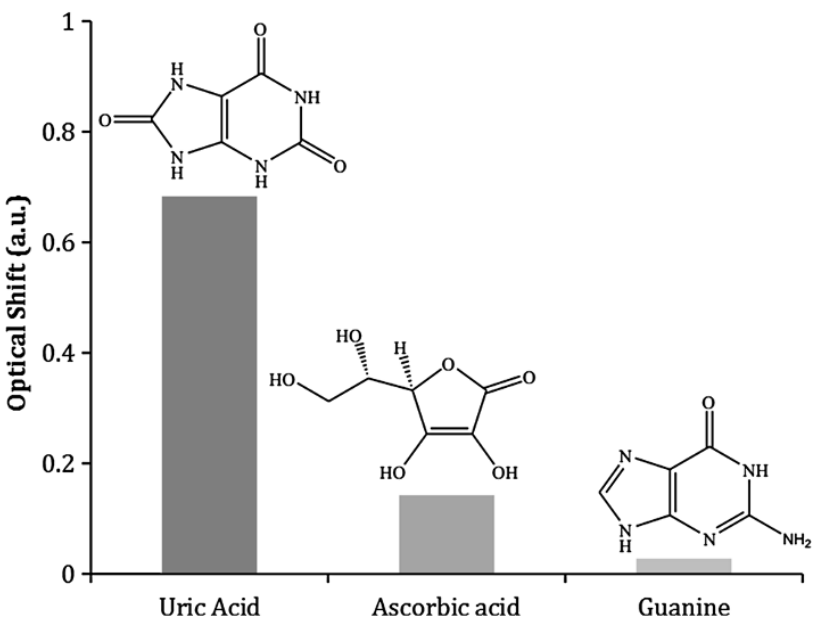

Fig. 6 Response of imprinted titania nanoparticles for uric acid (template), ascorbic acid (interfering compound), and guanine (structurally related) at equimolar concentrations

MINPs and NINPs for the same concentration of uric acid. It is obvious that the response of imprinted titania nanoparticles is about six times higher than non-imprinted ones. This suggests that NINPs can be taken as reference thus, to compensate all of the non-specific binding interactions.

Selectivity studies

In addition, we carried out selectivity experiments by immersing imprinted titania nanoparticles in ascorbic acid and guanine solutions of equimolar concentration, and the results are depicted in Fig. 6. It can be noticed that molecularly imprinted titania nanoparticles show higher binding affinity toward uric acid as compared to ascorbic acid and guanine. The ascorbic acid is considered as interfering compound in uric acid analysis, whereas the guanine is a purine and has somewhat similar structure to uric acid. The high optical shift is attributed to the successful imprinting of uric acid, i.e. the presence of uric acid-specific binding sites within imprinted titania nanoparticles' network. These results suggest that uric acid imprinted titania nanoparticles can perform efficiently in complex mixtures, and their binding is preferential toward uric acid even if closely related substances or interfering species are present in these mixtures.

\section{Comparison}

Table 2 gives a comparison of the results obtained in this study with reports published previously during the last few years (Patel et al. 2009; Sun et al. 2011; Khasanah et al. 2012; Wang et al. 2013). It is evident that uric acid imprinted titania nanoparticles, which can be produced rapidly using one-step synthetic procedure reported herein, offer the advantages of high sensitivity and selectivity, and 


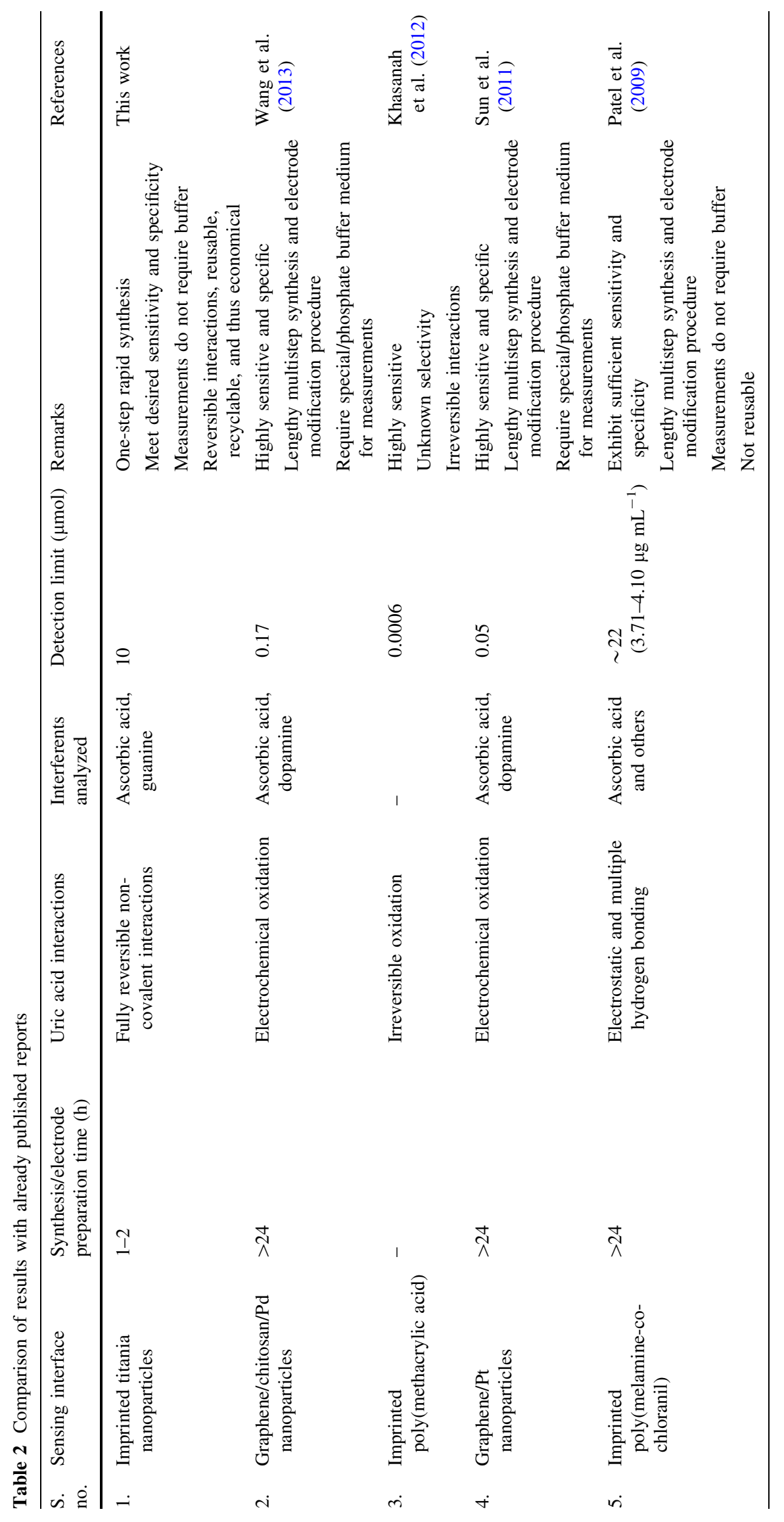


reversible non-covalent interactions with the analyte (uric acid). The latter makes them more useful as sensing interface due to their reusability and recyclability with minimum loss in their sensing properties, thus providing a low-cost method for repeated uric acid detection and assay measurements.

\section{Conclusions}

In this work, molecularly imprinted titania nanoparticles (MINPs) are developed for the sensitive and selective recognition of uric acid. Preliminary characterization of titania by AFM, SEM and FTIR indicated the formation of uniformly distributed nanoparticles with effective imprinting characteristics. The results of static adsorption capacity suggest that the binding capability of MINPs increases linearly with the increasing concentration of uric acid and the tested concentration range is comparable with the normal to elevated levels of uric acid in blood serum. The nonimprinted particles (NINPs) are considered as control (reference) as they showed negligible effect in comparison to imprinted titania nanoparticles. In addition, the interfering substances and structurally related compounds such as ascorbic acid and guanine showed significantly lower binding with MINPs that confirms the adequate selectivity of imprinted titania nanoparticles. Thus, imprinted titania nanoparticles could be integrated with a suitable transducer system in future for developing cost effective biomedical diagnostics for real-time monitoring of uric acid at trace levels in complex biological fluids.

Acknowledgments Authors thank Higher Education Commission (HEC) of Pakistan for providing the startup Grant No. PM-IPFP/ HRD/HEC/2011/0583.

Open Access This article is distributed under the terms of the Creative Commons Attribution License which permits any use, distribution, and reproduction in any medium, provided the original author(s) and the source are credited.

\section{References}

Andersson LI (2000) Molecular imprinting: developments and applications in the analytical chemistry field. J Chromatogr B Biomed Sci App 745:3-13

Ansell RJ, Mosbach K (1998) Magnetic molecularly imprinted polymer beads for drug radioligand binding assay. Analyst 123:1611-1616

Chen Z, Zhao R, Shangguan D, Liu G (2005) Preparation and evaluation of uniform-sized molecularly imprinted polymer beads used for the separation of sulfamethazine. Biomed Chromatogr 19:533-538. doi:10.1002/bmc.476

Chen L, Xu S, Li J (2011) Recent advances in molecular imprinting technology: current status, challenges and highlighted applications. Chem Soc Rev 40:2922-2942
Dickert FL, Hayden O (1999) Molecular imprinting in chemical sensing. TrAC Trends Anal Chem 18:192-199

Hilt JZ, Byrne ME (2004) Configurational biomimesis in drug delivery: molecular imprinting of biologically significant molecules. Adv Drug Deliv Rev 56:1599-1620

Horcas I, Fernández R, Gómez-Rodríguez JM et al (2007) WSXM: a software for scanning probe microscopy and a tool for nanotechnology. Rev Sci Instr 78:013705. doi:10.1063/1. 2432410

Iqbal N, Afzal A (2013) Imprinted polyurethane-gold nanoparticle composite films for rapid mass-sensitive detection of organic vapors. Sci Adv Mater 5:939-946. doi:10.1166/sam.2013.1542

Kaur H, Halliwell B (1990) Action of biologically-relevant oxidizing species upon uric acid. Identification of uric acid oxidation products. Chem Biol Interact 73:235-247

Kempe M, Mosbach K (1995) Molecular imprinting used for chiral separations. J Chromatogr A 694:3-13

Khasanah M, Mudasir AK, Sugiharto E (2012) Development of uric acid sensor based on molecularly imprinted polymethacrylic acid-modified hanging mercury drop electrode. J Chem Chem Eng 6:209-214

Kryscio DR, Peppas NA (2009) Mimicking biological delivery through feedback-controlled drug release systems based on molecular imprinting. AIChE J 55:1311-1324

Latif U, Mujahid A, Afzal A et al (2011) Dual and tetraelectrode QCMs using imprinted polymers as receptors for ions and neutral analytes. Anal Bioanal Chem 400:2507-2515

Lieberzeit PA, Afzal A, Glanzing G, Dickert FL (2007) Molecularly imprinted sol-gel nanoparticles for mass-sensitive engine oil degradation sensing. Anal Bioanal Chem 389:441-446

Maxwell SRJ, Thomason H, Sandler D et al (1997) Antioxidant status in patients with uncomplicated insulin-dependent and noninsulin-dependent diabetes mellitus. Eur $\mathrm{J}$ Clin Invest 27:484-490

Mohan J (2004) Organic spectroscopy: principles and applications. CRC Press, USA

Mosbach K (1994) Molecular imprinting. Trends Biochem Sci 19:9-14

Mujahid A, Afzal A, Glanzing G et al (2010a) Imprinted sol-gel materials for monitoring degradation products in automotive oils by shear transverse wave. Anal Chim Acta 675:53-57. doi:10. 1016/j.aca.2010.07.005

Mujahid A, Lieberzeit PA, Dickert FL (2010b) Chemical sensors based on molecularly imprinted sol-gel materials. Materials 3:2196-2217

Mujahid A, Iqbal N, Afzal A (2013) Bioimprinting strategies: from soft lithography to biomimetic sensors and beyond. Biotechnol Adv 31:1435-1447. doi:10.1016/j.biotechadv.2013.06.008

Patel AK, Sharma PS, Prasad BB (2009) Electrochemical sensor for uric acid based on a molecularly imprinted polymer brush grafted to tetraethoxysilane derived sol-gel thin film graphite electrode. Mater Sci Eng C 29:1545-1553. doi:10.1016/j.msec. 2008.12.008

Pérez N, Whitcombe MJ, Vulfson EN (2001) Surface imprinting of cholesterol on submicrometer core-shell emulsion particles. Macromolecules 34:830-836

Piletsky SA, Turner NW, Laitenberger P (2006) Molecularly imprinted polymers in clinical diagnostics-Future potential and existing problems. Med Eng Phys 28:971-977

Poma A, Turner AP, Piletsky SA (2010) Advances in the manufacture of MIP nanoparticles. Trends Biotechnol 28:629-637

Ramström O, Mosbach K (1999) Synthesis and catalysis by molecularly imprinted materials. Curr Opin Chem Biol 3:759-764

Rubab Z, Afzal A, Siddiqi HM, Saeed S (2014) Augmenting thermal and mechanical properties of epoxy thermosets: the role of

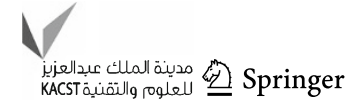


thermally-treated versus surface-modified $\mathrm{TiO}_{2}$ nanoparticles. Mater Express 4:54-64. doi:10.1166/mex.2014.1144

Sellergren B (2000) Molecularly imprinted polymers: man-made mimics of antibodies and their application in analytical chemistry. Elsevier, Amsterdam

Sellergren B, Allender CJ (2005) Molecularly imprinted polymers: a bridge to advanced drug delivery. Adv Drug Deliv Rev 57:1733-1741

Sun C-L, Lee H-H, Yang J-M, Wu C-C (2011) The simultaneous electrochemical detection of ascorbic acid, dopamine, and uric acid using graphene/size-selected Pt nanocomposites. Biosens Bioelectron 26:3450-3455. doi:10.1016/j.bios.2011.01.023

Vasapollo G, Sole RD, Mergola L et al (2011) Molecularly imprinted polymers: present and future prospective. Int $\mathrm{J}$ Mol Sci 12:5908-5945
Wang X, Wu M, Tang W et al (2013) Simultaneous electrochemical determination of ascorbic acid, dopamine and uric acid using a palladium nanoparticle/graphene/chitosan modified electrode. JElectroanal Chem 695:10-16. doi:10.1016/j.jelechem.2013.02.021

Wulff G (2002) Enzyme-like catalysis by molecularly imprinted polymers. Chem Rev 102:1-28

Ye L, Weiss R, Mosbach K (2000) Synthesis and characterization of molecularly imprinted microspheres. Macromolecules 33:8239-8245

Zhang L, Cheng G, Fu C (2003) Synthesis and characteristics of tyrosine imprinted beads via suspension polymerization. React Funct Polym 56:167-173

Zhang H, Ye L, Mosbach K (2006) Non-covalent molecular imprinting with emphasis on its application in separation and drug development. J Mol Recognit 19:248-259 